\title{
Aide à l'évaluation des risques aux postes de travail des radiologues industriels
}

\author{
B. LE GUEN ${ }^{1, \mathrm{a}}$, A. GARRIGOU ${ }^{2}$, G. ABÉLA ${ }^{3}$, M. FELTZINGER $^{4}$, P. RUEL $^{5}$, \\ P. BONAVENTURE ${ }^{1}$, J.-P. PATOUX ${ }^{6}$, P. ROENELLE ${ }^{7}$, \\ D. VERGNE $^{8}$, B. VAISSEAU ${ }^{9}$
}

RÉSUMÉ L'objectif de cet atelier du GT SFRP-COFREND a été de proposer une analyse des risques pour la réalisation future d'études de postes sur les tirs en milieu industriel (nucléaire et hors nucléaire). Le principe retenu a été de réaliser un découpage des différentes phases d'un chantier, en y associant à chaque étape les risques associés. Les examens et contrôles non destructifs (END et CND) sont des activités qui nécessitent une vigilance de tous les instants (Fig. 1) pour : s'assurer de la qualité du travail ; gérer les différents risques dont le risque d'exposition aux rayonnements ionisants; lutter contre des baisses d'attention liées aux formes de fatigue, aux conditions de travail, aux difficultés d'accès et dans certains cas au travail nocturne, etc. ; gérer les pressions temporelles (audits, surveillances...) ; gérer la co-activité pour éviter d'exposer d'autres professionnels; gérer le risque lié au milieu de travail (INB, présence de produits dangereux...). Le champ d'étude d'une analyse de risque couvre les métiers, les tâches et activités, les nuisances, et les risques... Le principe par la suite d'une étude de poste sera une approche descriptive et analytique de toutes les composantes de la réalité d'un travail donné, son organisation, son environnement, ses contraintes et moyens d'ajustement (Fig. 2). L'étude de poste s'appuiera sur des méthodes objectives d'observation, d'analyse de risque et de mesure (rapport du groupe de coordination étude de postes médecins du travail EDF). L'objectif de l'étude de poste est de lister l'ensemble des actions à mener pour garantir des conditions d'intervention et de travail acceptables afin de prévenir l'ensemble des risques.

ABSTRACT Guidelines for risks assessment at the workplaces of industrial radiologists.

The objective of this workshop of WP SFRP-COFREND was to propose an analysis of the risks for the future realization of studies of stations on Gamma Ray Non Destructive Testing (NDT) in industrial. The principle selected was to carry out a phase division various of a building site, by associating with each stage the associated risks with it. Gamma ray NDT are activities which require a vigilance of every moment for: to make

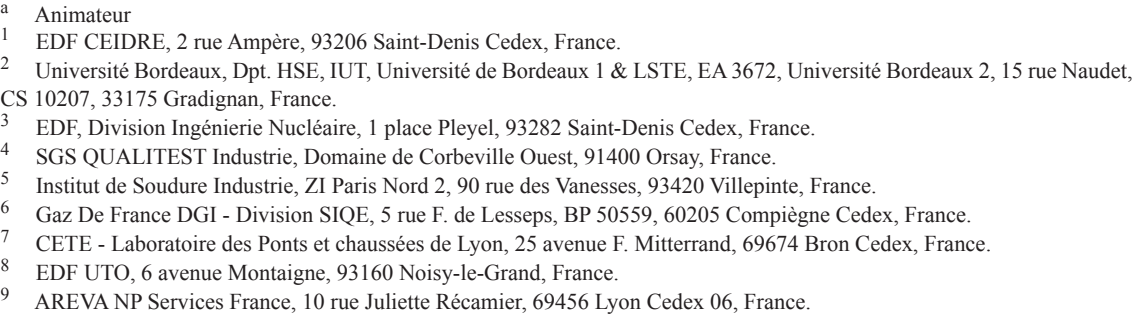


sure of the quality of work; to manage the various risks of which the risk of exposure to the ionizing radiations; to fight against falls of attention dependent on the forms of tiredness, the work conditions, the difficulties of access and in certain cases to night work, etc.; to manage the pressures temporal (Audits, monitorings...); to manage the coerciveness to avoid exposing other professionals, to manage the dependent risk in the middle of work (INB, presence of hazardous substances.). The field of study of an analysis of risk covers the trades, the tasks and activities, the harmful effects, and the risks...

Keywords: Workplace study / risk assessment / gamma ray Non Destructive Testing (NDT) / industrial NDT

\section{La description des activités}

\subsection{La préparation}

Les activités concernant les Examens Non Destructifs (END) doivent être définies par un programme détaillé des contrôles à effectuer (caractéristiques des éléments à contrôler (soudures, diamètre, nuances, épaisseur, dégradations etc.). Sur cette

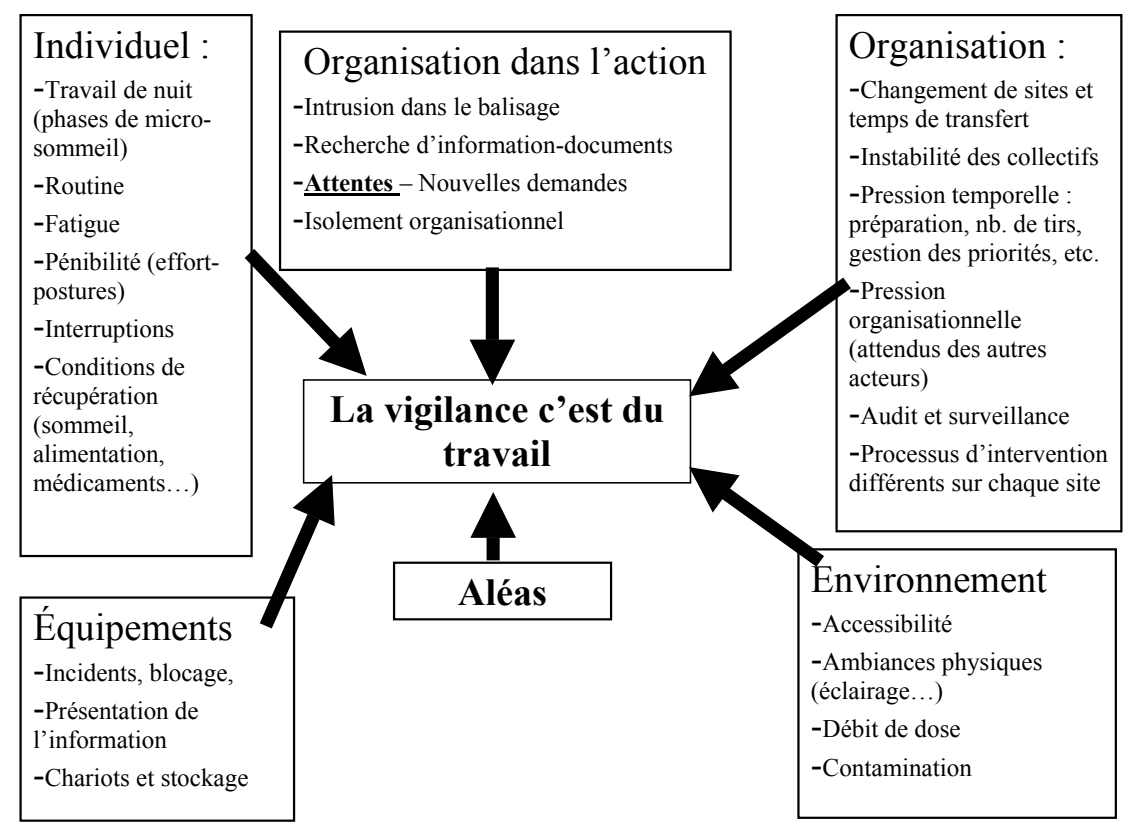

Figure 1 - Paramètres pouvant influencer la vigilance.

Parameters being able to influence vigilance. 


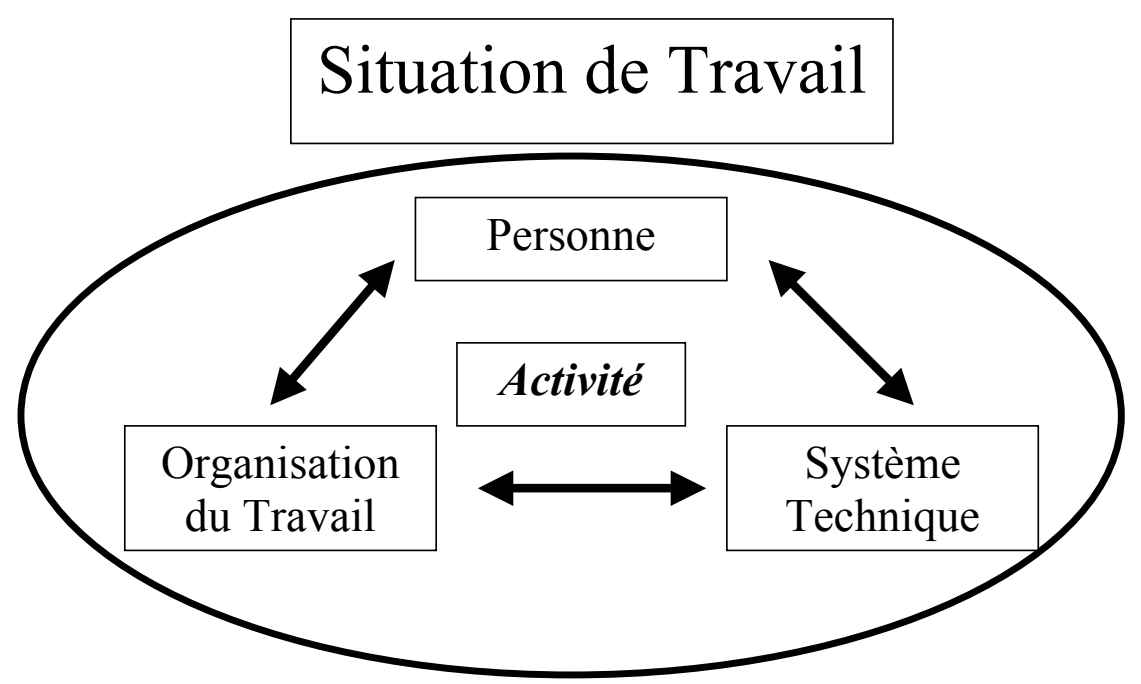

Figure 2 - Interrelation dans une situation de travail.

Interrelationship in a situation of work.

base l'entreprise d'END pourra définir les modes opératoires (choix de la source et de son activité, etc.) et la composition des équipes adaptés aux situations.

Le regroupement géographique des contrôles d'un même poste de travail constitue une bonne pratique qui permet de limiter la charge de travail en limitant les déplacements, et augmente ainsi l'efficacité de l'équipe.

Tout contrôle nécessite un permis de tir ou équivalent (fiches d'intervention, etc.) qui définit les moyens de prévention (définition d'un périmètre de sécurité, plan de balisage, estimation dosimétrique prévisionnelle individuelle et collective, identification des co-activités, identification des acteurs, etc.) identifiés à partir de l'analyse de risque (voir Fig. 3).

Ces documents sont préparés par l'entreprise qui effectue les END et transmis à l'entreprise utilisatrice, qui est responsable de la coordination des moyens de prévention (décret 92-158 ou équivalent et le décret travailleurs exposés 2003-296) et qui doit intégrer les formes de co-activité (autres métiers, dosimétrie induite, etc.). Avant toute réalisation ce document doit faire l'objet d'un accord formalisé, porté à la connaissance de tous les acteurs impliqués. Cet accord formalisé doit tenir compte des exigences de l'arrêté zonage (dosimétrie mensuelle et annuelle des personnels non classés ou du public présents à proximité de la zone d'opération). Le document signifiant cet accord peut être le « permis de tir » recommandé ci dessus. 


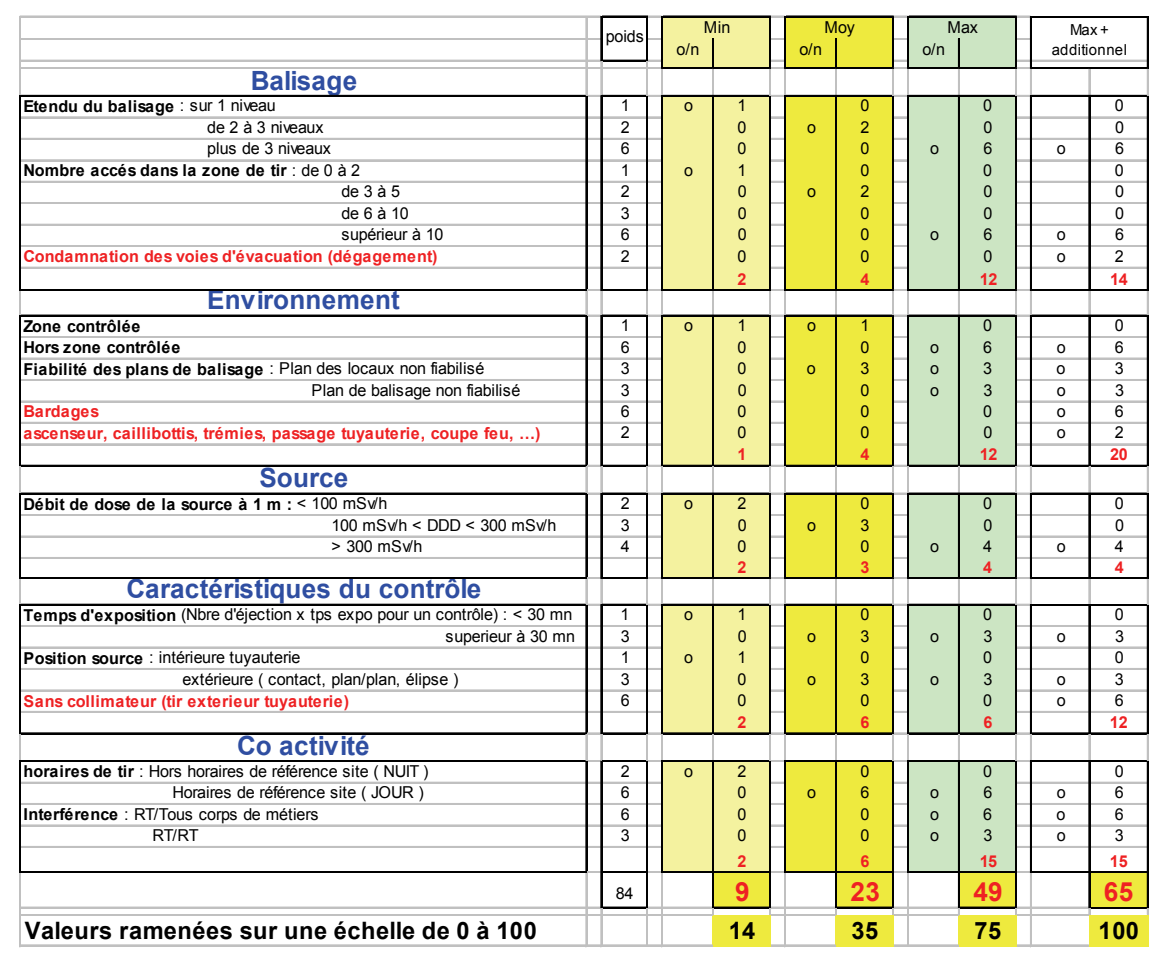

Figure 3 - Exemple d'analyse de risque d'un tir à EDF.

Example of weighing of a gamma ray NDT at EDF.

L'intégration des activités END dans le planning opérationnel actualisé en fonction des aléas rencontrés, est une nécessité pour garantir de bonnes conditions d'intervention. Pour exemple à EDF, un pesage de chaque tir est demandé et selon le résultat du pesage, des actions complémentaires sont mises en œuvre comme la prévision du contrôle externe du balisage (voir exemple : Fig. 3).

De même, dans le cadre de la formation, La sensibilisation régulière par la société intervenante de son personnel est impérative pour mettre en place une vraie culture de la radioprotection.

\subsection{La mise en place du chantier}

Un chantier, c'est beaucoup de matériel à transporter et à installer avant toute opération. Une bonne pratique peut être une simple check-list mise à disposition des intervenant qui permet de contrôler le matériel, d'éviter les oublis notamment lors de la phase de repli. 
Deux types très différents de matériel sont à disposition selon l'END et l'entreprise.

Pour la gammagraphie: un projecteur qui contient la source ; la gaine d'éjection, sa télécommande manuelle ou automatique et les accessoires d'irradiation (collimateur, embout d'irradiation, etc.).

Pour les rayons $X$ : un générateur $\mathrm{X}$ et son alimentation électrique, un écran en plomb disposé devant le fenêtre d'émission durant la phase de préchauffage ; les autres fournitures sont les films à exposer, des repères en plomb ou des marquages lumineux identifiant les contrôles, un système de fixation de la source, une balise lumineuse (type gyrophare ou lampe à éclat) à laisser à proximité de la source, des lampes à éclats oranges pour le balisage sont recommandés, du ruban de balisage et des trisecteurs (trèfle radioactif dont la couleur noir sur fond jaune est recommandée mais non obligatoire - norme NFM 60.103); des tendeurs, des sangles, des rubans autocollants, etc.

Le matériel de radioprotection devra être compatible avec le type de rayonnement et comprendre un radiamètre en plus des éléments de surveillance individuels (dosimètre actif à alarme sonore conforme à l'arrêté 26-10-2005 et un dosimètre type passif).

Un dossier est remis quotidiennement à chaque équipe. Ce dossier contient : les permis de tirs signés, les régimes de consignation, le plan de balisage et les documents administratifs (plan de prévention sous forme de consignes applicables), les ordres d'intervention, les procédures, une description des circuits (ISO) et un répertoire téléphonique.

Une bonne pratique est pour chaque activité de tir radio d'élaborer un dossier complet autoportant standardisé (CND et END) (permis de tirs, pesage du tir, plan de balisage...).

\subsection{Le transport du matériel}

Suivant les entreprises le stockage est assuré ou non par l'entreprise utilisatrice (donneur d'ordre). Dans le cas d'interventions de longue durée, il est recommandé que l'entreprise donneuse d'ordre assure l'entreposage des sources et du matériel des prestataires END et CND, quelque soit le donneur d'ordre (contractant ou sous-traitant de $1^{\mathrm{er}}$ ou $2^{\mathrm{e}}$ niveau).

Une bonne pratique est de prévoir ou fournir des moyens de transport adaptés au poids et au volume du matériel et ceci en nombre suffisant en cas de forte activité END (par exemple un nombre suffisant d'affichettes lumineuses). 


\subsection{Le balisage}

Le balisage est une activité qui peut sembler simple mais qui est fondamentale en terme de prévention. Dans la réalité, selon la configuration de l'espace à confiner en trois dimensions, du nombre d'entrées possibles, de la présence de dalles pleines ou bien de caillebotis, cette activité peut être complexe. Il est important de prévoir un temps suffisant pour sa réalisation. L'entreprise utilisatrice doit communiquer sur le balisage en terme de prévention de la même manière qu'elle communique par exemple lors des réunions de sécurité, sur le port du casque. Elle est le plus souvent réalisée par l'aide opérateur sous la responsabilité du chef d'équipe. Le balisage est de la responsabilité de l'équipe intervenante. En cas de contrôle de balisage, une bonne pratique est d'optimiser ce contrôle afin d'éviter les temps d'attente inutile.

\subsection{Mise en place du matériel}

Selon l'environnement de l'organe à contrôler (exemple de la présence d'éléments d'échafaudage, de la présence d'une électrovanne, etc.), la mise en place des films et des sources de rayonnement, peut devenir une tâche difficile. Afin de suivre les procédures de tirs, les opérateurs doivent mobiliser leurs connaissances techniques en radiographie (présence de filtre, focale, temps de pose, collimateur, etc.) mais aussi leurs savoir-faires professionnels pour s'adapter aux situations. L'emploi du collimateur est obligatoire sauf impossibilité technique justifiée. Hors industrie nucléaire, pour les générateurs $\mathrm{X}$, on privilégiera les faisceaux directionnels.

La coordination entre le contrôleur END et son aide est alors très importante, elle doit faire l'objet de nombreuses communications.

Le choix du positionnement de la gaine d'éjection et de la manivelle qui sert à éjecter est le résultat d'un compromis, il faut se protéger de la source tout en facilitant le contrôle visuel de la zone, et ce en évitant de plier la gaine. Cette étape peut être longue, dans le cas d'un contrôle sur un site EDF, nécessitant un temps de pose d'une minute, la mise en place des films, du GAM et de la canule ou du collimateur, peut durer près de 20 minutes.

\subsection{La dosimétrie ambiante}

Dans le cas de l'industrie nucléaire où les organes à contrôler sont situés dans des zones parfois à fort débit de dose, toutes les difficultés rencontrées lors de la mise en place des films et de l'embout d'irradiation vont rallonger les temps d'exposition de la personne aux rayonnements ionisants. Les coordinations avec les autres services pour mesurer les débits de dose ambiants, décalorifuger, installer des échafaudages et des protections biologiques, etc., sont alors prépondérantes. 
Le contrôle : le contrôleur n'est pas l'exécutant. Il doit notamment vérifier le balisage et son efficacité, mesurer le débit de dose au point de balisage, s'assurer de la traçabilité de toutes les opérations et identifier et assurer le traitement des écarts par rapport au plan de balisage et aux consignes de sécurité.

\subsection{La phase de tir}

À partir des variabilités rencontrées in situ, le contrôleur va tenter de suivre les procédures. Le calcul du temps de pose est réalisé en fonction de la densité requise, pour la zone concernée ; elle est indiquée sur la procédure applicable. En fonction du développement du premier film et surtout de sa densité, le contrôleur va ajuster in situ le temps de pose à la hausse ou bien à la baisse. Il faut souligner l'importance des communications entre le contrôleur in situ et la personne qui développe sur le laboratoire installé sur le site.

Lorsque les plages de densité exigée sont restrictives, le contrôleur doit attendre le résultat du développement du film qu'il vient de tirer. De nuit, ces temps d'attente peuvent être à l'origine d'une baisse de la vigilance.

De même, il est important de renforcer la communication vers les personnes sur le site pour éviter les franchissements de balisage par toute personne extérieure au chantier.

\subsection{La dosimétrie liée au tir}

L'exposition aux rayonnements ionisants est maximale lors des phases d'éjection et de réintroduction de la source dans le gammagraphe (GAM 80 ou 120 ou GR50). Le partage de la dosimétrie est indispensable, par exemple un seul opérateur manipule, l'autre devant rester en retrait.

Durant la phase de tir les moyens de protection sont basés : sur la distance par rapport à la source et l'utilisation des écrans naturels lorsque présents.

Tous les acteurs reconnaissent les risques présents lors de l'exposition du film ; les sources peuvent avoir une activité importante 2 à 4,4 tera Becquerels (environ 60 à 120 Curies, unité encore utilisée par les radiologues).

Lorsque la source est éjectée, les opérateurs doivent réaliser des mesures au niveau du balisage mis en œuvre, à l'aide d'un radiamètre (au début du tir). Les premiers tirs sont utilisés pour vérifier le débit d'équivalent de dose en limite de balisage (le tir d'essai n'est pas recommandé). Ces valeurs doivent être conformes à celles prévues par le permis de tir (Fig. 4). 


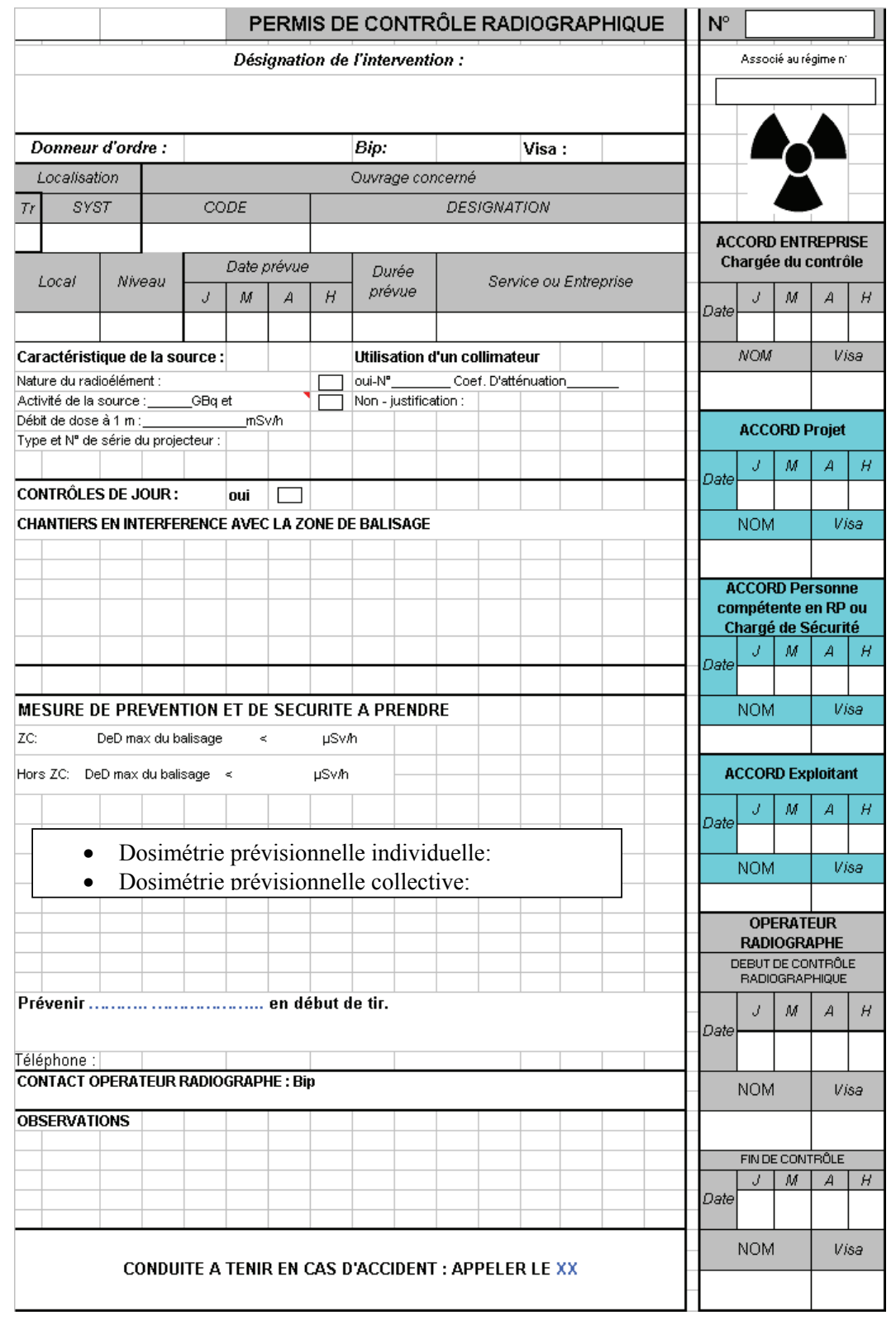

Figure 4 - Exemple de permis de contrôle radiographique.

Example of licence of gamma-ray NDT. 
Si le balisage n'est pas suffisant, l'opérateur en charge du balisage renforce ce dernier pour obtenir un débit de dose efficace inférieur ou égal à la catégorie du personnel susceptible d'intervenir à proximité du chantier.

Il est à noter que dans les conditions d'intervention réelles, la signalisation à voyant qui indique la présence du porte-source dans le GAM n'est pas toujours facilement accessibles ou visible. Ils ne sont visibles que de près, à condition d'avoir un éclairage suffisant. Dans les milieux à risques de contamination le GAM peut être protégé par un film de vinyl (tout comme la gaine d'éjection ou la canule), ce qui rend difficile la vision des informations présentes sur celui-ci.

La réglementation exige donc en complément, la vérification de l'absence de débit de dose à l'aide d'un radiamètre après chaque réintroduction du porte source ou de l'arrêt de l'émission (RX).

L'utilisation du compte tours de la télécommande et le comptage des tours de manivelle sont nécessaires pour vérifier :

- lors de l'éjection : l'arrivée dans l'embout d'irradiation (ou collimateur);

- lors de la réintégration : le retour du porte-source dans le projecteur (le son significatif du doigt d'obturateur peut être un autre signe de la réintégration complète du porte source).

L'utilisation du radiamètre atteste de ce risque mais ponctuellement, lorsque l'opérateur fait des mesures. Compte tenu de la gestion de l'incertitude que nous avons décrite ci-dessus, et des pressions qui sont ressenties par les opérateurs, un système d'alerte en continu (dosimètre actif individuel à alarme sonore) adapté aux rayonnements mesurés est obligatoire.

Du point de vue des opérateurs, des dosimètres à alarme peuvent être intéressants mais dans un milieu bruyant ils ne seront pas efficaces; un système de vibreur peut apporter une information complémentaire sous réserve que le dosimètre ne devienne un « stress » supplémentaire en cas de vibrations trop rapprochées si le niveau d'alarme est non adapté. (Nota : De nombreux dosimètres opérationnels ont trois principes de signalisation ou d'indication : la valeur numérique, le niveau sonore et l'affichage lumineux.)

\subsection{La phase de repli chantier}

Cette phase devra comprendre :

- la signalisation de la fin du tir de manière formelle et la sécurisation du chantier (retrait de la clef du gammagraphe ou du pupitre du générateur X) ;

- le retrait complet du balisage et du matériel de signalisation ; 
- le retrait des déchets produits (vinyl, etc.);

- le retour de la source dans le local prévu pour son stockage ;

- le transport de l'ensemble du matériel.

\section{Analyse des risques}

L'analyse des risques devra porter sur l'ensemble des risques présents : risques radiologiques, risques de chute, risques liés aux manutentions, risques psychosociaux, risques liés au travail de nuit, aux ambiances physiques, etc. Pour chacun de ces risques il sera important d'identifier leurs déterminants, qu'ils soient d'ordre technique, organisationnel ou humain (voir Tab. I). L'enjeu final en terme de prévention est de limiter ainsi toutes les formes d'atteintes à la vigilance des opérateurs.

L'identification des difficultés par phase de l'activité END fait apparaître les éléments suivants.

\subsection{Pendant la phase de préparation}

- Le temps minimum nécessaire de préparation du chantier, différent d'un chantier à un autre.

- Le délai nécessaire aux entreprises prestataires pour composer les équipes et ce en fonction des niveaux de compétences requises.Pour certains équipes, la contrainte de temps supplémentaire pour l'obtention des autorisations de transport et des périodes de chargement de sources par Cegelec.

- La prise en compte des déplacements inter sites des radiologues et de leurs périodes de repos.L'évaluation des risques liés aux situations incidentelles (risques différents entre un générateur $\mathrm{X}$ au repos et une source cobalt).

- La dosimétrie prévisionnelle individuelle et collective et gestion de la dose au niveau des équipes.

- Le choix du matériel et des modes opératoires adaptés à l'END demandé.

- La recherche des documents nécessaires dont les plans : importance de la fiabilité des plans de balisage selon une représentation standardisée et de la visite des locaux.

- La fourniture des consignes de sécurité applicables.

- La maintenance et la préparation du matériel nécessaire.

L'un des risques de l'organisation mise en place dans un dossier préparatoire dont le prescriptif est volumineux est une déclinaison plus sur la forme que sur le fond et le sens. L'implication et la co-responsabilité de tous les acteurs est fondamentale que ce soit pour:

- la coordination, la planification et la communication entre les différents acteurs en particulier en cas d'intervention nocturne où il est important 
d'organiser la liaison jour-nuit pour l'activité de tirs radios entre les prestataires et la société utilisatrice; ;'interface entre les différents acteurs ;

- une bonne préparation du chantier en amont avec si nécessaire vidange des circuits, décalorifugeage, échafaudage ;

- les risques liés à la gestion des aléas et la charge de travail liée à la gestion administrative.

\subsection{Pendant la phase de préparation sur site}

- la localisation du local source et des locaux de stockage du matériel et son accessibilité, en regard de la zone de tir ;

- les horaires d'intervention;

- les difficultés de communication entre les différents acteurs et métiers impliqués : les opérateurs peuvent rencontrer des problèmes pour joindre leurs interlocuteurs (développeur, bureau de consignation, ou salle de commande) soit pour des raisons d'ambiances sonores élevées à proximité du poste de téléphone, de téléphone défaillant, ou bien de lignes occupées. Ces problèmes de communication viennent renforcer l'incertitude ;

- l'affichage des tirs non adapté ;

- le transport des équipements (risques liés aux manutentions, de chute du personnel et du matériel) : il est important de souligner les difficultés concernant le transport et la manutention du matériel avec des risques de chutes, et des postures à risques dorsolombaire pour l'intervenant ;

- le risque lié au matériel manquant ou insuffisant ;

- le repérage des organes à contrôler ;

- la mise en place du balisage et son contrôle.

Le balisage doit permettre à toute personne extérieure au chantier de comprendre rapidement et clairement l'interdiction de franchissement. Le contrôle du balisage est systématique chez EDF (contrôle et point d'arrêt). Il est réalisé par sondage chez les autres industriels. Il est important que ce système reste vertueux et complémentaire. En particulier Il est demandé d'harmoniser les modalités d'accès à une zone de tir quelque soit le rôle hiérarchique ou réglementaire de la personne qui veut accéder au chantier.

Parmi les autres risques identifiables, on notera les risques liés à une baisse de la « vigilance» du radiologue, liés à un sentiment de déresponsabilisation du fait de la connaissance du contrôle du chantier, liés à l'attente de la disponibilité du vérificateur et aux dérives potentiels du temps d'intervention en cas de sous-traitance du contrôle (financièrement plus « rentable » pour le bénéficiaire en cas d'intervention longue). En conséquence les équipes responsables chargées du contrôle doivent être informées pour optimiser leur temps d'intervention. 
TABLEAU I

Exemple d'identification des risques associés et des barrières techniques, humaine (formation) ou organisationnelles.

Example of the associated risks and the technical, human or organisational countermeasures.

\begin{tabular}{|c|c|c|c|c|c|c|c|}
\hline Activités & Dangers & $\begin{array}{l}\text { Risques : } \\
F^{*} G\end{array}$ & $\begin{array}{l}\text { Situations } \\
\text { à risques }\end{array}$ & Cibles & $\begin{array}{l}\text { Barrières } \\
\text { techniques }\end{array}$ & $\begin{array}{l}\text { Barrières } \\
\text { humaines }\end{array}$ & $\begin{array}{c}\text { Barrières } \\
\text { organisationnelles }\end{array}$ \\
\hline $\begin{array}{l}\text { Transport } \\
\text { du } \\
\text { matériel }\end{array}$ & $\begin{array}{l}\text { Blessures } \\
\text { dorso- } \\
\text { lombaires }\end{array}$ & $\begin{array}{l}\text { Fréquence: } \\
\text { à chaque } \\
\text { début de } \\
\text { poste et fin } \\
\text { de poste } \\
\text { Gravité : } \\
\text { Importante }\end{array}$ & $\begin{array}{l}\text { Port } \\
\text { et } \\
\text { manutention } \\
\text { des } \\
\text { équipements }\end{array}$ & $\begin{array}{l}\text { Les } \\
\text { radiologues }\end{array}$ & $\begin{array}{l}\text { Chariot adapté } \\
\text { au matériel et à } \\
\text { l'environnement }\end{array}$ & $\begin{array}{l}\text { Sensibilisation } \\
\text { sur le thème : } \\
\text { " "Geste } \\
\text { et posture» }\end{array}$ & $\begin{array}{l}\text { Réservation } \\
\text { et mise } \\
\text { à disposition } \\
\text { des chariots }\end{array}$ \\
\hline Tir & Irradiations & $\begin{array}{l}\text { Fréquence : } \\
\text { à chaque tir } \\
\text { Gravité : } \\
\text { très } \\
\text { importante }\end{array}$ & $\begin{array}{l}\text { Entrée } \\
\text { dans le } \\
\text { balisage } \\
\text { pendant } \\
\text { le tir }\end{array}$ & $\begin{array}{l}\text { Les } \\
\text { radiologues } \\
\text { et les autres } \\
\text { travailleurs }\end{array}$ & $\begin{array}{l}\text { Dosimètre à } \\
\text { alarmes } \\
\text { Balise à alarme } \\
\text { Balisage, etc. }\end{array}$ & $\begin{array}{l}\text { Développement } \\
\text { des } \\
\text { compétences }\end{array}$ & $\begin{array}{l}\text { Mise en place } \\
\text { de formation } \\
\text { Procédures } \\
\text { adaptées et } \\
\text { utilisables }\end{array}$ \\
\hline
\end{tabular}

Pendant la mise en place du film et des accessoires d'irradiation : lors de la fixation de la canule ou des films, des postures déséquilibrées à risque peuvent s'imposer aux opérateurs, par exemple parfois à l'intérieur d'une canalisation, ou bien exigeant de se coucher sur le sol avec des risques de contamination. Enfin l'oubli ou l'inattention peuvent être détermines par des facteurs techniques, humains ou organisationnels (voir schéma 1).

\section{Les risques liés à l'environnement :}

- l'accessibilité à la zone de travail et à la zone de repli des radiologues ;

- les ambiances de travail (éclairage, température ambiante, bruit, atmosphère à risques (chimique, explosive - par exemple dans la pétrochimie ou le gaz), débit de dose ambiant (intervention en zone contrôlée);

- l'éventage éventuel des circuits.

\subsection{Pendant la phase de tir}

Les risques sont liés :

- aux attentes liées à l'obtention des autorisations de tir, au fonctionnement des installations à l'analyse du résultat du tir : le radiologue vit une situation d'incertitude forte, où il ne sait pas si le résultat du tir va être satisfaisant. Cette réponse peut prendre au moins une heure. Dans certains cas, si le contrôleur a des tirs à proximité il peut alors commencer à les préparer, voire à «tirer» de nouveau (la encore on retrouve les mêmes risques liés à l'oubli ou l'inattention);

- à la lutte contre l'endormissement, avec maintien de la vigilance pour faire face aux exigences de l'activité ; 
- aux positionnement des radiologues vis-à-vis de la source au moment du tir ;

- à la surveillance du balisage pour éviter des risques liés à des intrusions sur le chantier;

- à l'évolution des ambiances de travail (conditions météo, etc.) ;

- aux aléas : interruptions, modifications fortuites de l'environnement, fuites, etc. ;

- aux interférences liée à la co-activité et à la présence d'autres chantiers.

On peut noter l'écart qui existe entre la durée d'un chantier et la durée pendant laquelle la source a été éjectée. À partir d'un exemple, il a été observé que pour un chantier d'une durée de près de $4 \mathrm{~h}$ portant sur 6 films, la source n'a été éjectée que 6 fois une minute. Cela signifie en termes de risques d'exposition que les tireurs n'ont été potentiellement affectés que $6 \mathrm{mn}$ sur près de $4 \mathrm{~h}$ de travail ; et réellement affectés que lors de $6 \times 2$ phases de transit qui n'excèdent pas 3 secondes.

\subsection{Pendant la phase de repli}

Les risques sont liés à :

- l'information de l'arrêt des tirs ;

- l'oubli ou l'inattention déterminées par des facteurs techniques, humains ou organisationnels (voir schéma 1);

- la lutte contre l'endormissement, avec maintien de la vigilance pour faire face aux exigences de l'activité ;

- la gestion des déchets ;

- la récupération et le transport du matériel (importance de la check-list).

Une des bonnes pratiques pour s'assurer que les points clés de la prise en compte de la spécificité des contrôles radiographiques par les donneurs d'ordre sont appliqués dans les différentes industries est de désigner un coordinateur, superviseur unique pour l'ensemble des contrôles radiographiques. Il permet de prendre en compte la nécessité de préparer les interventions, d'assurer un appui en temps réel aux intervenants, et de constituer un retour d'expérience utile.

Les activités de ce correspondant pourraient être décrites par une lettre de mission (ou équivalent) et seront surtout mentionnées dans le plan de prévention.

Les activités principales de ce coordinateur seraient alors de :

- Fournir les conditions de réussite des activités : accessibilité des organes, éclairage, vidange, planification en fonction des travaux amonts tels que les décalorifugeages.

- Accueillir les intervenants à leur arrivée sur le site, organiser au mieux l'intervention (il s'agit des intervenants de terrain, pas uniquement de leur 
hiérarchie) : préciser le rôle des acteurs, établir un premier contact, commenter les notes d'application, le niveau d'exigence du site, prendre connaissance des bonnes pratiques des autres industriels.

- Procéder au retour d'expérience (REX) de l'intervention lors du départ du site des intervenants : procéder à le remontée des bonnes pratiques, et plus généralement examiner le vécu de l'intervention par les intervenants de terrain.

- Effectuer au moins une visite de chantier accompagné d'un représentant du service prévention des risques du site. L'objet de cette visite est de faire partager la spécificité du métier de radiologue à des personnes chargées d'exercer des contrôles de terrain, et d'harmoniser le niveau d'exigence.

- Déterminer en prenant l'appui d'experts si nécessaire les contrôles pour lesquels l'utilisation du collimateur n'est pas possible. L'utilisation du collimateur constitue la pratique normale. C'est sa non-utilisation qui doit être justifiée.

- Identifier les zones à contrôler, et fournir des schémas explicites. Les plans « iso » constituent une bonne référence, qui est aussi utile aux calorifugeurs, aux échafaudeurs, et plus généralement aux logisticiens.

- Informer les équipes de quart de l'installation de la spécificité du métier de radiologue. Il est nécessaire d'aborder par exemple l'importance des lignages permettant une vidange complète des tuyauteries, les installations qui seront inaccessibles pendant les contrôles radio, le rôle des équipes de quart dans la détection des écarts.

- Assurer une présence de terrain suffisante pour que le passage de consignes entre les équipes en journée et de nuit soit fait, et que les intervenants de nuit disposent d'un appui de la part de l'entreprise utilisatrice. Cet appui peut aussi être utile pour gérer des conflits de planning entre différentes entreprises intervenantes.

- Traiter et diffuser les enseignements tirés de toutes les situations de type « presque accident » radiologique.

- Aider l'employeur à planifier le travail de contrôle par zones de travail proches. Cette pratique vise à limiter les transports et les manutentions au cours d'un même poste de travail.

\section{Conclusion}

Le radiologue peut se sentir «noyé » dans une organisation très complexe (de l'obtention du permis de tir, des levées des points d'arrêt, de la vérification du balisage etc.). Cette organisation a un impact important sur son environnement. Dans le temps total d'intervention, la préparation et la phase de repli sont donc deux phases primordiales pour éviter tout incident, l'activité réelle de tir étant, elle, très réduite. Du fait de la vigilance demandée à chaque étape du processus, la condition physique des intervenants est un facteur essentiel. 
Les examens et contrôles non destructifs (END et CND) sont des activités complexes qui nécessitent la participation d'acteurs multiples aux responsabilités différentes appartenant tant à la société intervenante qu'à l'entreprise utilisatrice et la mise en place d'une vraie culture de radioprotection. La maîtrise de l'activité de tirs industriels nécessite attention et rigueur de tous les acteurs à chaque instant. La pratique ne peut être performante que si chacun est conscient de sa responsabilité.

Remerciements. Les auteurs tiennent à remercier les intervenants anonymes à la journées EDF «Forum sur les tirs gammagraphiques sur un site nucléaire » du 20 décembre 2006 qui par leur participation aux tables rondes ont permis d'enrichir cette réflexion. 